\title{
Conocimiento y preparación de los odontólogos mexicanos ante la pandemia por COVID-19.
}

\section{Knowledge and preparation of Mexican dentists facing the COVID-19 pandemic.}

\author{
Enrique Netzahualcóyotl Cavazos-López,* Diana Antonieta Flores-Flores, ${ }^{\ddagger}$ Alicia Rumayor-Piña, ${ }^{\S}$ Patricia Torres-Reyes,॥ \\ Óscar Rodríguez-Villarreal," Beatriz Catalina Aldape-Barrios**
}

\section{RESUMEN}

Introducción: La pandemia a la que nos enfrentamos globalmente ha impactado de manera importante a la práctica odontológica, ya que, debido al uso de aerosoles, es una profesión de alto riesgo de transmisión de COVID-19. Objetivos: Describir los conocimientos generales sobre el SARS-CoV-2, recomendaciones y medidas de protección en una muestra de odontólogos mexicanos. Material y métodos: Se realizó un estudio descriptivo, transversal utilizando un instrumento de evaluación que se distribuyó en línea a instituciones públicas, privadas y asociaciones dentales mexicanas. Los resultados fueron analizados por medio de estadística descriptiva. Resultados: La encuesta fue respondida por 1,286 odontólogos, el 73.1\% reportó haberse capacitado recientemente acerca del SARS-CoV-2. En su mayoría conocen sobre grupos de riesgo, transmisión, características clínicas, y recomendaciones generales. La mayoría se encuentran atendiendo exclusivamente urgencias y demuestran conocimiento acerca de los equipos de protección personal y del protocolo de atención durante la pandemia. Conclusiones: Los odontólogos mexicanos cuentan con los conocimientos sobre las generalidades del SARS-CoV-2, así como sobre los protocolos de atención y equipos de protección personal para la atención de pacientes durante la pandemia por COVID-19.

Palabras clave: COVID-19, SARS-CoV-2, odontología, conocimientos.

\section{ABSTRACT}

Introduction: The current pandemic that we are facing globally has had a significant impact on dental practice, due to the use of aerosols it is a profession with high risk of CoViD 19 transmission. Objective: To describe the general knowledge about SARS-CoV-2, as well as on the recommendations and personal protective equipment in a sample of Mexican dentists. Material and methods: A descriptive, transversal study was carried out through an evaluation instrument distributed on-line during 20 consecutive days to several dental professionals of private and public institutions and Mexican dental associations. Results were analyzed using descriptive statistics. Results: 1,286 Mexican dental professionals completed the survey, $73.1 \%$ indicated having received training about SARS-CoV-2 and they mostly know about the individuals at higher risk, transmission, clinical features and general recommendations. Most of the dentists are exclusively attending urgencies as pulpal pain, abscess and dental traumatisms. Knowledge regarding the use of personal protective equipment was demonstrated, as well as the protocol of care during the pandemic. Conclusions: Mexican dentists have the knowledge on the generalities of SARS-CoV-2, on care protocols and protective measures for patient care during the CoViD-19 pandemic.

Keywords: COVID-19, SARS-CoV-2, dentistry, knowledge.

\section{INTRODUCCIÓN}

$\mathrm{E}^{\mathrm{n}}$ n diciembre del 2019 se reportó a las oficinas de la Organización Mundial de la Salud (OMS) en China, la presencia de una neumonía atípica en la Ciudad de
Wuhan, provincia de Hubei, en enero los científicos aislaron al nuevo coronavirus SARS-CoV-2 (síndrome agudo respiratorio severo coronavirus 2) y el día 30 del mismo mes del 2020 la OMS reportó este brote como una «Emergencia de Salud Pública de Carácter Interna-

\footnotetext{
* Maestría en Ciencias Odontológicas. Facultad de Odontología Unidad Saltillo. Universidad Autónoma de Coahuila. México.

‡ Maestría en Estomatología Pediátrica. Facultad de Odontología Unidad Torreón. Universidad Autónoma de Coahuila. México.

§ Doctorado en Estomatopatología. Facultad de Odontología Unidad Saltillo. Universidad Autónoma de Coahuila. México.

॥ Maestría en Ciencia y Tecnología de Enzimas. Facultad de Odontología Unidad Saltillo. Universidad Autónoma de Coahuila. México.

ๆ Maestría en Ciencias Odontológicas. Facultad de Odontología Unidad Torreón. Universidad Autónoma de Coahuila. México.

** Maestría en Patología Bucal. Facultad de Odontología. Universidad Nacional Autónoma de México, CU. México.

Recibido: 13 Mayo 2020. Aceptado para publicación: 19 Mayo 2020.
} 
cional», denominándolo COVID-19. ${ }^{1}$ La OMS reporta 3,267,184 casos confirmados, 229,971 defunciones y específicamente en la región de las Américas 1,340,591 casos confirmados y 72,196 defunciones hasta la fecha en que se escribe este artículo. ${ }^{2}$ En México, el primer caso confirmado se reportó ante las autoridades de la Secretaría de Salud el 27 de febrero del 2020, y el reporte al 5 de mayo sobre la situación de la enfermedad COVID-19 señala que hay 22,088 casos confirmados, y 2,061 defunciones. ${ }^{3}$

EI SARS-CoV-2 es un coronavirus de cadena de ARN de la generación b, rodeado por una membrana bilipídica con una serie de glicoproteínas, simulando espinas que utiliza para unirse con el receptor de la membrana de las células que producen la enzima convertidora de la angiotensina 2 (ECA2) que se encuentran en el sistema renal, gastrointestinal, corazón y pulmones. ${ }^{4} \mathrm{La}$ evidencia disponible hasta el momento propone que la fisiopatología es una respuesta inmune exagerada de los alveolos pulmonares por el incremento de la interleucina 6 (IL-6) ${ }^{5}$ y una posterior disminución en la producción de linfocitos $B$, resultando en un síndrome agudo respiratorio severo. ${ }^{6}$ Aunado a esto, los hallazgos recientes reportan una coagulación intravascular diseminada, interviniendo elementos celulares y plasmáticos de las primeras etapas de la respuesta inmune. ${ }^{7}$ Los síntomas comunes son fiebre, tos seca y dificultad para respirar, ${ }^{8}$ aunque las últimas actualizaciones del CDC incluyen otros como dolor de garganta, cefalea, mialgia, diarrea, escalofríos, temblores y pérdida del olfato o el gusto. 9,10

El método de transmisión del SARS-CoV-2 es por medio de gotículas expulsadas por personas contagiadas al toser o estornudar, contacto con secreciones de pacientes enfermos de COVID-19, así como superficies, equipo e instrumental contaminado. ${ }^{11,12}$

Se encuentran en riesgo de manera particular los profesionales de la odontología y sus pacientes, que, debido al uso continuo de equipo e instrumental generador de aerosoles, están expuestos a microorganismos patógenos y virus presentes en la boca y en el tracto respiratorio, este aerosol puede ser inhalado, entrar en contacto con piel o membranas mucosas y permanecer en las superficies del consultorio. La evidencia científica reporta que el SARS-CoV-2 se mantiene viable en aerosoles durante tres horas, y permanece más tiempo en acero inoxidable que en plástico, conservando su viabilidad hasta 72 horas después. ${ }^{13}$

En México, este aspecto se encuentra regulado por la Norma Oficial Mexicana NOM-013-SSA2-2015, Para la prevención y control de enfermedades bucodentales, donde se abordan todos los procedimientos de control de infecciones y seguridad del paciente durante la atención dental. ${ }^{14}$ En fechas recientes la Secretaría de Salud, las Secretarías de Salud Estatales, la Asociación Dental Mexicana (ADM) y las diferentes asociaciones de especialidades dentales han hecho una serie de recomendaciones para la atención de la práctica dental durante el periodo «Emergencia Sanitaria por Causas de Fuerza Mayor» que declaró el Gobierno de México a partir del 30 de marzo del 2020. ${ }^{15-17}$

En general, las recomendaciones incluyen medidas preventivas universales como: 1 ) lavado de manos con agua de 40 a 60 segundos y jabón o alcohol gel, 2) al toser o estornudar utilizar la parte interna del codo y usar pañuelos desechables, 3) evitar saludarse de mano, beso o abrazo y 4) no tocarse las mucosas de ojos, nariz y boca, y para la atención de pacientes, dar prioridad a las consultas de urgencia, y posponer las consultas ordinarias hasta nuevo aviso, realizar toma de temperatura a todos los pacientes como un proceso rutinario, así como protocolos de atención para los pacientes con y sin síntomas en los últimos 14 días. $^{18}$

Dichas recomendaciones no difieren mucho de las reportadas en otros países latinoamericanos, ${ }^{19} \mathrm{ni}$ de otros continentes. ${ }^{20-22}$ El reto es el alcance de estas capacitaciones y conocimientos a los profesionales de la odontología, así como su aplicación práctica durante esta pandemia para evitar que el consultorio dental se convierta en un punto de riesgo para el dentista, el equipo de trabajo y los pacientes. Por lo tanto, el objetivo de este trabajo es determinar el nivel de preparación en cuanto a conocimientos y medidas generales sobre el SARS-CoV-2 en odontólogos mexicanos.

\section{MATERIAL Y MÉTODOS}

Se realizó un estudio descriptivo y transversal que consistió en la aplicación de un instrumento de autollenado elaborado a partir de una revisión de la literatura relacionada con las características generales y clínicas de COVID-19, así como de las recomendaciones nacionales e internacionales para la atención dental durante la pandemia. ${ }^{23-26}$ El instrumento de medición se llevó a cabo por medio de una encuesta de opción múltiple en español utilizando Microsoft Forms ${ }^{\circledR}$ con 21 preguntas relacionadas a la práctica odontológica, estatus laboral durante la pandemia de COVID-19, percepción de urgencia dental, capacitación actual y conocimientos generales de COVID-19, así como 
de las recomendaciones publicadas para la práctica odontológica y su aplicación durante la pandemia de COVID-19. Para finalizar, se preguntó si conocían a un odontólogo enfermo de COVID-19 y se pidió agregar un comentario a la encuesta. Se debe aclarar que había variables de selección única y otras politómicas. Al inicio y al final se aclaró que las respuestas se registraban como anónimas y de esta manera asegurar la confidencialidad de los resultados conseguidos en el estudio. Según el registro del Instituto Mexicano para la Competitividad A.C., hasta el 2019 se encuentran ejerciendo en México 117,266 odontólogos. ${ }^{27}$ Se utilizó la fórmula de tamaño de muestra infinita, con un nivel de confianza del $99 \%$ y un margen de error de $4 \%$, con lo que se obtuvo una muestra representativa de 1,031 individuos. ${ }^{28}$

La encuesta fue distribuida por medio de la aplicación WhatsApp o de Facebook o durante un periodo de 20 días (13 de abril al 2 de mayo) a la Asociación Dental Mexicana, Coordinación de Salud Bucal de la Secretaría de Salud del Estado de Coahuila, presidentes de colegios y asociaciones dentales nacionales, estatales y locales, presidentes de asociaciones de especialidades dentales, directores de facultades e institutos de enseñanza odontológica, así como a coordinadores de postgrados de especialidades dentales de diversas universidades e institutos de México; con la intención de que fuera distribuida y contestada por sus integrantes. Los resultados se tabularon en Microsoft Excel ${ }^{\circledR}$ y se analizaron por medio de estadística descriptiva por medio de distribución de frecuencia y porcentaje.

\section{RESULTADOS}

La encuesta fue contestada por un total de 1,286 odontólogos mexicanos. La muestra fue integrada en su mayoría por mujeres, con un rango de edad de entre 20 y 40 años. Con respecto a los años de práctica odontológica, hubo una ligera prevalencia de aquéllos que tenían una práctica de entre uno y 10 años. La gran mayoría de los encuestados se encuentran inmersos en el sector privado y practican odontología general. Cabe destacar que el sector profesional y área de práctica son variables politómicas, por lo tanto, la suma de n será mayor que el número de muestra (Tabla 1).

El $73.1 \%$ de los encuestados respondieron que se habían capacitado recientemente en prevención y control de infecciones causadas por el SARS-CoV-2 (945), las fuentes de capacitación más referidas incluyeron instituciones de salud gubernamentales y artículos y revistas científicas. La mayoría de los odontólogos coincidió en

\section{Tabla 1: Características sociodemográficas $(\mathrm{N}=1,286)$.}

\begin{tabular}{lrr} 
Variable & $\mathrm{n}$ & $\%$ \\
\hline Género & & \\
$\quad$ Femenino & 839 & 65.0 \\
Masculino & 447 & 35.0 \\
Edad (años) & & \\
20-29 & 372 & 29.0 \\
$30-39$ & 338 & 26.3 \\
40-49 & 266 & 20.7 \\
$50-59$ & 187 & 14.6 \\
$>$ 60 & 123 & 9.4 \\
Tiempo de práctica (años) & & \\
1-10 & 489 & 38.0 \\
11-20 & 387 & 30.0 \\
> 20 & 410 & 31.8 \\
Sector profesional & & \\
Privado & 1,116 & 86.7 \\
Universitario & 324 & 25.1 \\
Público & 208 & 16.1 \\
Militar & 10 & 0.7 \\
Área de práctica & & \\
Odontología General & 826 & 64.2 \\
Ortodoncia & 340 & 26.4 \\
Endodoncia & 226 & 17.5 \\
Odontología Infantil & 188 & 14.6 \\
Prostodoncia & 175 & 13.6 \\
Periodoncia & 121 & 9.4 \\
Cirugía Maxilofacial & 53 & 4.1 \\
Otra & 48 & 3.7 \\
\hline
\end{tabular}

que los individuos considerados de alto riesgo para contagiarse por SARS-CoV-2 son adultos mayores de 60 años, los que padecen enfermedades crónico-degenerativas como diabetes y los que se encuentran en estado de inmunosupresión. Un 1.1\% declaró desconocer esta información. El 85.5\% de los encuestados respondieron que el periodo de incubación del SARS-CoV-2 era de uno a 14 días y referente a los síntomas característicos de COVID-19 más del 90\% de los odontólogos consideraron a la fiebre (mayor a $38^{\circ} \mathrm{C}$ ), disnea y tos seca. Con relación al conocimiento de las formas de transmisión del SARSCoV-2 más del $90 \%$ de los encuestados refirieron que es a través de gotículas expulsadas por personas infectadas y contacto con superficies y equipos contaminados. En el conocimiento de las medidas generales para evitar el contagio por SARS-CoV-2, más del 98\% de los encuestados 
contestaron conocer las recomendaciones. Un pequeño grupo $(0.3 \%)$ desconoce los síntomas característicos de la enfermedad y su modo de transmisión, así como las recomendaciones de prevención (Tabla 2).

\begin{tabular}{|c|c|c|}
\hline \multicolumn{3}{|c|}{$\begin{array}{l}\text { Tabla 2: Conocimientos acerca de SARS-CoV-2 y } \\
\text { COVID-19 }(\mathrm{N}=1,286) .\end{array}$} \\
\hline Variable & $\mathrm{n}$ & $\%$ \\
\hline \multicolumn{3}{|l|}{ Capacitación } \\
\hline Sí & 945 & 73.1 \\
\hline No & 341 & 26.5 \\
\hline \multicolumn{3}{|l|}{ Fuente de capacitación (n = 945) } \\
\hline Instituciones de salud gubernamentales & 521 & 55.1 \\
\hline Artículos y revistas científicos & 439 & 46.4 \\
\hline Organización Mundial de la Salud & 365 & 38.6 \\
\hline \multicolumn{3}{|l|}{ Grupos con alto riesgo de contagio } \\
\hline Adultos $>60$ años & 1,232 & 95.8 \\
\hline Diabéticos & 1,199 & 93.2 \\
\hline Inmunosuprimidos & 1,195 & 92.9 \\
\hline Hipertensos & 1,142 & 88.8 \\
\hline Desconozco & 13 & 1.1 \\
\hline \multicolumn{3}{|l|}{ Periodo de incubación (días) } \\
\hline 1-14 & 1,100 & 85.5 \\
\hline \multicolumn{3}{|l|}{ Síntomas característicos } \\
\hline Fiebre $>38{ }^{\circ} \mathrm{C}$ & 1,273 & 98.9 \\
\hline Disnea & 1,272 & 98.9 \\
\hline Tos & 1,188 & 92.3 \\
\hline Cefalea & 1,028 & 79.9 \\
\hline Malestar general & 807 & 62.7 \\
\hline Se puede presentar sin síntomas & 792 & 61.5 \\
\hline Diarrea & 431 & 33.5 \\
\hline Náuseas y vómito & 166 & 12.9 \\
\hline Desconozco & 4 & 0.3 \\
\hline \multicolumn{3}{|l|}{ Formas de transmisión } \\
\hline Gotículas & 1,241 & 96.5 \\
\hline Contacto con superficies contaminadas & 1,209 & 94.0 \\
\hline Transmisión a partir de animales & 307 & 23.8 \\
\hline Desconozco & 4 & 0.3 \\
\hline \multicolumn{3}{|l|}{ Recomendaciones generales } \\
\hline Higiene de manos & 1,281 & 99.6 \\
\hline $\begin{array}{l}\text { Distancia mínima de 1-2 metros } \\
\text { entre personas }\end{array}$ & 1,278 & 99.3 \\
\hline Evitar saludar de manos o de beso & 1,274 & 99.0 \\
\hline Estornudar en la parte interna del codo & 1,243 & 96.6 \\
\hline Evitar tocarse nariz, cara y boca & 1,265 & 98.3 \\
\hline Desconozco & 4 & 0.3 \\
\hline
\end{tabular}

Un 61\% de odontólogos mexicanos está únicamente ofreciendo atención de urgencia, 33\% no están realizando ningún tipo de procedimiento y un $6 \%$ está llevando su consulta con normalidad. El dolor asociado a pulpitis reversible e irreversible, absceso periapical, trauma dental y celulitis fueron las principales atenciones consideradas como urgencias. Es importante destacar que más de la mitad de los encuestados (56\%) no consideraron como urgencia la biopsia por sospecha de patología maligna y $1.1 \%$ desconocían qué procedimientos se consideran urgencia.

Dentro de la sección sobre equipo de protección personal (EPP), más del 90\% de los encuestados respondieron que contaban y utilizarán principalmente guantes, careta protectora, lentes, gorro y bata desechable. Respecto al tipo de máscara de protección, $77.8 \%$ respondieron que utilizarían cubrebocas quirúrgico y un $62.5 \%$ cubrebocas N95. Las principales medidas consideradas por los odontólogos como protocolo de atención de pacientes en el consultorio dental incluyeron la desinfección y esterilización del instrumental (96.1\%), higiene de manos (antes y después del tratamiento) (94.8\%), y la evaluación previa del paciente vía telefónica (interrogatorio de síntomas característicos) (94.3\%) (Tabla 3).

El 87 y $71 \%$ de los encuestados respondieron tener conocimiento del método correcto de lavado de manos con jabón y desinfección con alcohol, respectivamente, como lo recomienda la OMS. Finalmente, 95\% de los encuestados reportaron no conocer odontólogos padeciendo COVID-19.

\section{DISCUSIÓN}

Ante la pandemia por COVID-19, el conocimiento de medidas de prevención generales y de prevención para infecciones cruzadas en el odontólogo es crucial. Los hallazgos de este estudio señalan que los dentistas mexicanos se han capacitado en proporciones similares por medios gubernamentales, artículos científicos, OMS, y las asociaciones dentales nacionales, estatales y locales, quienes se han encargado de distribuir acertadamente la información requerida.

En relación con los conocimientos generales del SARS-CoV-2 y la enfermedad COVID-19, se evidencia la capacitación de los odontólogos en el reconocimiento de los síntomas principales, es decir, tos, dificultad para respirar y fiebre, el porcentaje de odontólogos que tienen conocimiento sobre los síntomas menos comunes o que han sido reportados en estudios de caso individuales es menor. 


\section{Tabla 3: Conocimiento sobre la preparación para la atención dental.}

\begin{tabular}{|c|c|c|}
\hline Variable & $\mathrm{n}$ & $\%$ \\
\hline \multicolumn{3}{|l|}{ Atención dental } \\
\hline Normal & 74 & 6.0 \\
\hline Urgencias & 790 & 61.0 \\
\hline Ninguna & 422 & 33.0 \\
\hline \multicolumn{3}{|l|}{ Atenciones consideradas urgencias dentales } \\
\hline Dolor asociado a pulpitis reversible e irreversible & 1,092 & 84.9 \\
\hline Absceso periapical & 916 & 71.2 \\
\hline Trauma dental & 833 & 64.7 \\
\hline Celulitis & 832 & 64.6 \\
\hline Luxación de ATM que necesite reducción & 744 & 57.8 \\
\hline Pericoronitis & 669 & 52.0 \\
\hline Fractura de una restauración, comprometiendo función masticatoria & 588 & 45.7 \\
\hline Biopsia por sospecha de patología maligna & 567 & 44.0 \\
\hline Retiro de sutura de procedimiento quirúrgico reciente & 461 & 35.8 \\
\hline Desajuste de prótesis & 219 & 17.0 \\
\hline Ajuste de aparatología de ortopedia maxilofacial & 112 & 8.7 \\
\hline Ajuste ortodóncico & 90 & 6.9 \\
\hline Desconocimiento del concepto considerado como urgencia & 15 & 1.1 \\
\hline \multicolumn{3}{|l|}{ Equipo de protección personal } \\
\hline Guantes & 1,276 & 99.2 \\
\hline Careta protectora & 1,220 & 94.8 \\
\hline Gafas o lentes de protección & 1,183 & 91.9 \\
\hline Gorro & 1,173 & 91.2 \\
\hline Bata desechable & 1,170 & 90.9 \\
\hline Mascarilla quirúrgica o cubrebocas & 1,001 & 77.8 \\
\hline Mascarilla N95 & 805 & 62.5 \\
\hline Botas quirúrgicas desechables & 609 & 47.3 \\
\hline \multicolumn{3}{|l|}{ Protocolo para la atención de pacientes } \\
\hline Desinfección y esterilización del instrumental & 1,224 & 96.1 \\
\hline Higiene de manos & 1,220 & 94.8 \\
\hline Previo interrogatorio de síntomas (vía telefónica) & 1,213 & 94.3 \\
\hline Recomendar acudir a consulta solo o con un padre o tutor & 1,171 & 91 \\
\hline Manejo correcto de residuos biológico-infecciosos & 1,146 & 89.1 \\
\hline Sanitización y desinfección del área clínica & 1,107 & 86 \\
\hline Antisepsia bucal previa al procedimiento & 895 & 69.5 \\
\hline Aislamiento absoluto & 881 & 68.5 \\
\hline Medición de la temperatura & 598 & 45.8 \\
\hline Pieza de alta velocidad antirretracción & 380 & 29.5 \\
\hline
\end{tabular}

Referente a las recomendaciones para la atención dental, es conveniente señalar que la definición del término urgencia dental está basada en juicio clínico personal y debe realizarse de forma individual con cada paciente tomando en cuenta síntomas, naturale- za del padecimiento, entre otros. Es importante aclarar la diferencia entre procedimientos de emergencia y urgencia dental. La Asociación Dental Americana determina procedimientos de emergencia aquéllos que representen un riesgo potencial para la vida y 
que requieran tratamiento inmediato como sangrado continuo, alivio de dolor severo o infección no controlada con aumento de volumen intra o extraoral con riesgo de comprometer la vía aérea o traumatismo que involucre huesos faciales. Una urgencia dental se centra en el manejo de condiciones que requieran atención inmediata para alivio de dolor y con riesgo de infección, principalmente dolor severo asociado con patología pulpar, pericoronitis, absceso o trauma, aquellas restauraciones o prótesis desajustadas que generen irritación de tejidos blandos y sospecha de patología maligna. ${ }^{29}$ Los resultados de esta encuesta fueron concordantes con lo descrito en la literatura, con mayor porcentaje de selección aquéllos que presentan sintomatología dolorosa, y con el menor porcentaje los ajustes de ortodoncia y de aparatología ortopédica maxilofacial. Los ajustes de ortodoncia no son considerados como procedimientos urgentes, se recomienda mantenerse en contacto con el paciente de forma remota y evitar el uso de elementos activos que generen fuerzas, así como reservar la atención exclusiva a necesidades que no se puedan solucionar de forma remota. ${ }^{21}$ Es importante y grave que casi el $60 \%$ de los odontólogos mexicanos no consideraron la realización de biopsia por sospecha de patología maligna como urgencia. Está indicado que lesiones con alta sospecha de malignidad deben tener prioridad para realización de biopsia, confirmación de diagnóstico y canalización a centros especializados, ya que son entidades que poseen alto riesgo de mortalidad si no son diagnosticadas o si lo son en etapas avanzadas, siendo la más común el carcinoma oral de células escamosas. ${ }^{30}$ Finalmente, el concepto de urgencia dental debe estructurarse de forma concisa y concreta, y difundirse para que ayude al odontólogo mexicano a establecer las pautas de atención durante esta pandemia. En la literatura se reportan las urgencias endodónticas y quirúrgicas como las más recurrentes. ${ }^{19}$

Por su parte, el conocimiento de los EPP y los protocolos de atención dental que usa o considera usar el dentista mexicano durante esta pandemia concuerda en la mayoría con las recomendaciones nacionales e internacionales, a excepción del uso de máscara en el que en su mayoría (77.8\%) seleccionaron que usarían cubrebocas quirúrgico y solamente un $62.5 \%$ seleccionó a la mascarilla N95, siendo que ésta última es la indicada por la OMS para utilizarse en procedimientos que generen aerosol. ${ }^{31}$ Menos del 50\% de odontólogos señalaron que incluirían la medición de la temperatura como medida rutinaria, considerando que este aspecto ya se ha comunicado de manera pública y oficial en el país. Esta preparación y conocimiento se ha reflejado en el bajo porcentaje de contagios de odontólogos por COVID-19. Según las cifras oficiales, de los 1,934 profesionales de la salud enfermos por COVID-19, el 1\% es en odontólogos. ${ }^{32}$ En este estudio, 5\% de los odontólogos señalaron conocer a otro odontólogo con contagio por COVID-19, teniendo en cuenta que no se determinó si su contagio fue debido a su práctica profesional y con la limitación de un posible sesgo en este porcentaje, ya que posiblemente varios odontólogos encuestados pudieran señalar una misma persona.

\section{CONCLUSIONES}

Este panorama de la odontología en México durante la pandemia mundial puede dejar en claro que la difusión de protocolos de atención y medidas de protección de infecciones ha brindado frutos. Todos los profesionales de la salud deberán tomar la responsabilidad de evitar contagios por el SARS-CoV-2, y en especial los odontólogos. Con los resultados obtenidos, se requiere la preparación de Universidades, facultades de odontología, asociaciones dentales y organizaciones gubernamentales, ya que se enfrentan a un nuevo desafío. Regulaciones en abastecimiento y costos de los EPP fomentarán un mejor apego a los protocolos y recomendaciones establecidas, así como la creación de información, manuales o formatos prácticos y objetivos basados en la evidencia científica que ayude a los académicos, alumnos, equipo de trabajo y odontólogos de todos los ámbitos laborales a prepararse con los nuevos protocolos y plan de acción dental para el periodo postpandémico COVID-19 en México.

\section{AGRADECIMIENTOS}

Un especial agradecimiento a las y los presidentes, coordinadores y directores de las diversas Instituciones Gubernamentales, Asociaciones, Colegios, Universidades e Institutos de Enseñanza que apoyaron para la realización de esta investigación.

Asociación Dental Mexicana, Secretaría de Salud del Estado de Coahuila, Gobierno Municipal de Saltillo, Colegio de Cirujanos Dentistas del Estado de Coahuila A.C., Colegio de Cirujanos Dentistas de la Laguna, A.C., Colegio de Cirujanos Dentistas del Sur de Tamaulipas A.C., Colegio de Cirujanos Dentistas del Puerto de Veracruz A.C., Colegio de Cirujanos Dentistas de Irapuato 
A.C., Colegio de Dentistas de Hermosillo A.C., Colegio Dental de Ciudad Obregón A.C., Colegio de Odontología Infantil de Saltillo A.C., Colegio de Odontólogos de Michoacán A.C., Asociación Sinaloense de Especialistas en Odontopediatría A.C., Asociación Odontológica de Querétaro, Colegio de Cirujanos Dentistas A.C., Asociación Dental de León Colegio de Cirujanos Dentistas A.C., Asociación Mexicana de Endodoncia Colegio de Especialistas A.C. y filiales, Sociedad Odontológica Jaliscience, A.C., Facultad de Odontología Unidad Saltillo. Universidad Autónoma de Coahuila, Facultad de Odontología Unidad Torreón. Universidad Autónoma de Coahuila, Facultad de Odontología. Universidad Nacional Autónoma de México, CU, Universidad de Medicina Oral de Coahuila, Instituto Profesional Odontológico Contemporáneo.

\section{BIBLIOGRAFÍA}

1. Mahase E. China coronavirus: WHO declares international emergency as death toll exceeds 200. BMJ. 2020; 368: m408.

2. World Health Organization. Coronavirus disease 2019 (COVID-19): situation report, 103. World Health Organization; 2020. Available in: https://apps.who.int/iris/handle/10665/3320573.

3. Gobierno de México. Secretaría de Salud. Subsecretaría de Prevención y Promoción de la Salud. Comunicado Técnico Diario COVID-19 México (2/05/2020). 2020. Disponible en: https://www. gob.mx/salud/prensa/nuevo-coronavirus-en-el-mundo-covid-19comunicado-tecnico-diario-241474? idiom $=\mathrm{es}$

4. Cascella M, Rajnik M, Cuomo A et al. Features, evaluation and treatment coronavirus (COVID-19) [Updated 2020 Apr 6]. In: StatPearls [Internet]. Treasure Island (FL): StatPearls Publishing; 2020. Available from: https://www.ncbi.nlm.nih.gov/books/ NBK554776/

5. Moore JB, June $\mathrm{CH}$. Cytokine release syndrome in severe COVID-19. Science. 2020; 368: 473-474.

6. Di Gennaro F, Pizzol D, Marotta C, Antunes M, Racalbuto V, Veronese $\mathrm{N}$ et al. Coronavirus diseases (COVID-19) current status and future perspectives: a narrative review. Int J Environ Res Public Health. 2020; 17 (8): E2690.

7. Lillicrap D. Disseminated intravascular coagulation in patients with 2019-nCoV pneumonia. J Thromb Haemost. 2020; 18 (4): 786-787.

8. Kamps BS, Hoffmann C. COVID Reference. Steinhäuser Verlag; 2020.

9. Del Rio C, Malani PN. 2019 Novel Coronavirus-important information for clinicians [published online ahead of print, 2020 Feb 5]. JAMA. 2020; 10.1001/jama.2020.1490.

10. Centers for Disease Control and Prevention. Coronavirus Disease 2019. Symptoms. Available in: https://www.cdc.gov/ coronavirus/2019-ncov/symptoms-testing/symptoms.html. Published 2020.

11. Meng L, Hua F, Bian Z. Coronavirus disease 2019 (COVID-19): emerging and future challenges for dental and oral medicine. J Dent Res. 2020; 99 (5): 481-487.

12. Huang C, Wang Y, Li X, Ren L, Zhao J, Hu Y et al. Clinical features of patients infected with 2019 novel coronavirus in Wuhan, China. Lancet. 2020; 395 (10223): 497-506.
13. van Doremalen N, Bushmaker T, Morris DH, Holbrook MG, Gamble A, Williamson BN et al. Aerosol and Surface Stability of SARS-CoV-2 as Compared with SARS-CoV-1. N Engl J Med. 2020; 382 (16): 1564-1567.

14. Norma Oficial Mexicana NOM-013-SSA2-2015, Para la prevención y control de enfermedades. 2016. pp. 1-30. Disponible en: http://www.dof.gob.mx/nota_detalle.php?codigo $=5462039 \& f e c$ ha $=23 / 11 / 2016$

15. LARR. Asociación Dental Mexicana. Comunicado COVID-2019. 2020. Disponible en: https://www.facebook.com/ADMorgmx/ photos/pcb.656822201552383/10157688069566858/?type $=3$ \&theater

16. Robles D, Rodríguez Casanovas HJ. El COVID-19 y la consulta dental: información y consejos. 2020. Disponible en: https:// gacetadental.com/2020/03/el-covid-19-y-la-consulta-dentalinformacion-y-consejos-95967/

17. Torre Martínez HH. Asociación Mexicana de Ortodoncia. Comunicado Oficial. 2020.

18. Gobierno de México. Recomendaciones para la práctica odontológica. 2020. Disponible en: https://coronavirus. gob.mx/wp-content/uploads/2020/04/Recomendaciones_ Odontologicas_16042020.pdf

19. Sigua-Rodríguez EA, Bernal-Pérez JL, Lantana-Flores AG, SánchezRomero C, Rodríguez-Chessa J, Haidar ZS et al. ¿COVID-19 y la Odontología?: una revisión de las recomendaciones y perspectivas para Latinoamérica. Int J Odontostomatol. 2020; 14 (3): 299-309.

20. Alharbi A, Alharbi S, Alqaidi S. Guidelines for dental care provision during the COVID-19 pandemic. Saudi Dent J. 2020; 32 (4): 181-186.

21. Caprioglio A, Pizzetti GB, Zecca PA, Fastuca R, Maino G, Nanda R. Management of orthodontic emergencies during 2019-NCOV. Prog Orthod. 2020; 21: 10.

22. Centers for Disease Control and Prevention. Dental Settings. Interim Infection Prevention and Control Guidance for Dental Settings During the COVID-19 Response. Available in: https:// www.cdc.gov/coronavirus/2019-ncov/hcp/dental-settings.html. Published 2020.

23. Organización Mundial de la Salud. Prevención y control de infecciones $(\mathrm{PCl})$ causadas por el nuevo coronavirus (COVID-19). Módulo 2: Nuevo coronavirus (COVID-19) características epidemiológicas, factores de riesgo, definiciones y sintomatología. 2020.

24. Organización Mundial de la Salud. Prevención y control de infecciones (PCl) causadas por el nuevo coronavirus (COVID-19). Módulo 3: PCl en el contexto de la COVID-19. Precauciones habituales, precauciones basadas en el modo de transmisión y recomendaciones específicas para la COVID-19. 2020.

25. Organización Panamericana de la Salud. Requerimientos para uso de equipos de protección personal (EPP) para el nuevo coronavirus (2019-nCoV) en establecimientos de salud. Disponible en: https:// iris.paho.org/handle/10665.2/51976

26. Peng X, Xu X, Li Y, Cheng L, Zhou X, Ren B. Transmission routes of 2019-nCoV and controls in dental practice. Int J Oral Sci. 2020;12 (1): 9 .

27. Instituto Mexicano para la Competitividad. Estomatología y odontología. Published 2018. Disponible en: https://imco.org.mx/ comparacarreras/carrera/713

28. Habib A, Johargy A, Mahmood K, Humma H. Design and determination of the sample size in medical research. IOSR J Dent Med Sci. 2014; 13 (5): 21-31.

29. American Dental Association. What constitutes a dental emergency? Available in: https://success.ada.org/ /media/CPS/Files/Open\%20 
Files/ADA_COVID19_Dental_Emergency_DDS.pdf. Updated $3 / 31 / 20$

30. Ajudarte LM, Santos Silva AR, VargasPA, Kowalski LP. Virtual assistance in oral medicine for prioritizing oral cancer diagnosis during the COVID-19 pandemic. Oral Surg Oral Med Oral Pathol Oral Radiol. 2020. [Epub ahead of print]

31. Coulthard P. Dentistry and coronavirus (COVID-19) - moral decision-making. Br Dent J. 2020; 228 (7): 503-505.

32. Ortiz A, Perla M. México llega a mil 221 muertes y suma 12 mil 872 contagios. Periódico El Universal. Disponible en: https://www. eluniversal.com.mx/nacion/mexico-llega-mil-221-muertes-y-suma12-mil-872-contagios. Published 2020.

\section{Correspondencia:}

\section{Alicia Rumayor Piña}

Avenida Dra. Cuquita Cepeda de Dávila s/n,

Col. Adolfo López Mateos,

25125, Saltillo, Coahuila.

Tel: (01 844) 434-0030

E-mail: aliciarumayor@uadec.edu.mx 